$D$-Reactive polyarthritis, including Reiter's syndrome, develops following infection and is a mild to severe migratory asymmetrical aseptic polyarthritis mainly affecting the large joints. ${ }^{7}$ The condition most commonly follows gastroenteritis caused by Salmonella, Shigella, Campylobacter, and Yersinia, or urogenital infection caused by Chlamydia trachomatis. ${ }^{7}$ The diagnosis is preferably confirmed by isolation of the causative micro-organism. Post-chlamydial arthralgia, however, can be successfully diagnosed by demonstration of the group (CFT) antibody and confirmed by detection of the type specific antibody by immunofluorescence. Yersinia serology is also available but isolation of the organism is more satisfactory.

$E$ - Reactive migratory arthritis exhibiting little joint swelling also occurs in Lyme disease. The diagnosis is difficult because of the six or more weeks delay in appearance of detectable antibody. ${ }^{8}$ Serology is only undertaken when there is a clinical history suggestive of erythema migrans or possible exposure to tick bites.

$F$-In the past brucellosis was an established cause of arthritis but the cases reported annually to the Communicable Disease Surveillance Centre, London, have declined from 271 in 1972 to 16 in 1992, with nine infections acquired abroad. Therefore, investigation for Brucella agglutinins is only undertaken when there is a history suggestive of exposure.

$G-$ No travel history was obtained from any of the cases in this series. In the past, however, polyarthritis following Ross River Valley Fever, Ockelbo, West Nile, and Sindbis viral infections has been diagnosed in this laboratory after travel abroad. ${ }^{9}$ To ensure such cases are not overlooked, a travel component was incorporated in the algorithm.
An awareness that in certain geographical areas rodent borne, aerosol spread virus infections can cause polyarthralgia led to the recent observations in this country of the symptom complex reported in association with hantavirus infection. ${ }^{10}$ The infection is relevant in rural areas particularly when there is a history of possible exposure to rodents.

The application of this algorithm to the investigation of arthralgia will assist in selection of the appropriate tests while keeping unhelpful tests to a minimum. A total of 753 individual tests were carried out in this series. If the rubella $\operatorname{IgM}(£ 7.76)^{*}$, the monolatex test $(£ 0.99)^{*}$ and screening for viral infection $(£ 7.44)^{*}$ are omitted from the initial battery of tests, 335 $(44 \cdot 4 \%)$ fewer investigations are required with a possible saving of $£ 740$.

1 Smith James W. Infectious arthritis. In: Mandell GL, Douglas RG, Bennet JE, eds. Principles and practice of infectious diseases. 3rd edn. Edinburgh: Churchill Livingstone, 1985: diseases. 3 rd

911-18.

Pinals RS. Polyarthritis and fever. N Engl f Med 1994;330: 769-74.

3 Jones JW, Pether JVS, Frost RWP. Human parvovirus B19. Hard to differentiate from infectious mononucleosis. $B M \mathcal{F}$ 1994;308:595.

4 Ross PW. Streptococcal diseases. In: Smith GR, Easmon CSF, eds. Topley $\mathcal{G}$ Wilson Principles of bacteriology, virology and immunity. 3. Bacterial diseases. London: Edward Arnold, 1990:246-8.

5 Lee PR. Arthritis and rubella. BM7 1962;ii:925.

6 Reid DM, Reid TMS, Brown T, Rennie JAN, Eastmond CJ. Human parvovirus associated arthritis: A clinical and laboratory description. Lancet 1985;i:422-5.

laboratory description. Lancet 1985;i:422-5.
Svenungsson B. Reactive arthritis. BMf 1994;308:671-2.

7 Svenungsson B. Reactive arthritis. BMf 1994;308:671-2.

8 Steere AC. Lyme disease. N Engl f Med 1989;321:586-96. caused by an arbovirus. $\mathcal{F}$ Rheumatol $1983 ; 10$ (Suppl):34-6.

10 Pether JVS, Lloyd G. The clinical spectrum of human hantavirus infection in Somerset, UK. Epidemiol Infect 1993;111:171-5.

* These prices were obtained from a preliminary costing exercise.

\title{
Fatal adenovirus 32 infection in a bone marrow transplant recipient
}

\author{
Department of \\ Paediatric Pathology, \\ Royal Children's \\ Hospital, \\ Bristol \\ A K Charles \\ H J Porter \\ Bone Marrow \\ Transplant Unit \\ A Oakhill \\ Public Health \\ Laboratory, \\ Bristol \\ E O Caul \\ Correspondence to: \\ Dr A Charles, \\ Department of Paediatric \\ Pathology, \\ St Michael's Hospital, \\ Southwell Street, \\ Bristol BS2 8EG. \\ Accepted for publication \\ 18 January 1995
}

\author{
A K Charles, E O Caul, H J Porter, A Oakhill
}

\begin{abstract}
A case of disseminated adenovirus type 32 infection causing severe hepatitis, gastrointestinal ulceration and also with respiratory involvement is reported in a bone marrow transplant recipient. Typical viral inclusions were seen in the postmortem histological sections and adenovirus infection was confirmed using in situ hybridisation and isolation of adenovirus type 32 from separate organs at necropsy. This is the first case in which adenovirus 32 was the cause of fatal disseminated dis-
\end{abstract}

ease in a bone marrow transplant recipient.

(f Clin Pathol 1995;48:779-781)

Keywords: Adenovirus, bone marrow transplant, in situ hybridisation.

Adenovirus infections are well recognised as a cause of serious morbidity and mortality in immunocompromised patients ${ }^{1}$ and have been reported in renal, ${ }^{2}$ liver, ${ }^{3}$ and bone marrow transplant recipients. ${ }^{45}$ The complications include hepatitis, pancreatitis, ${ }^{6}$ bowel in- 


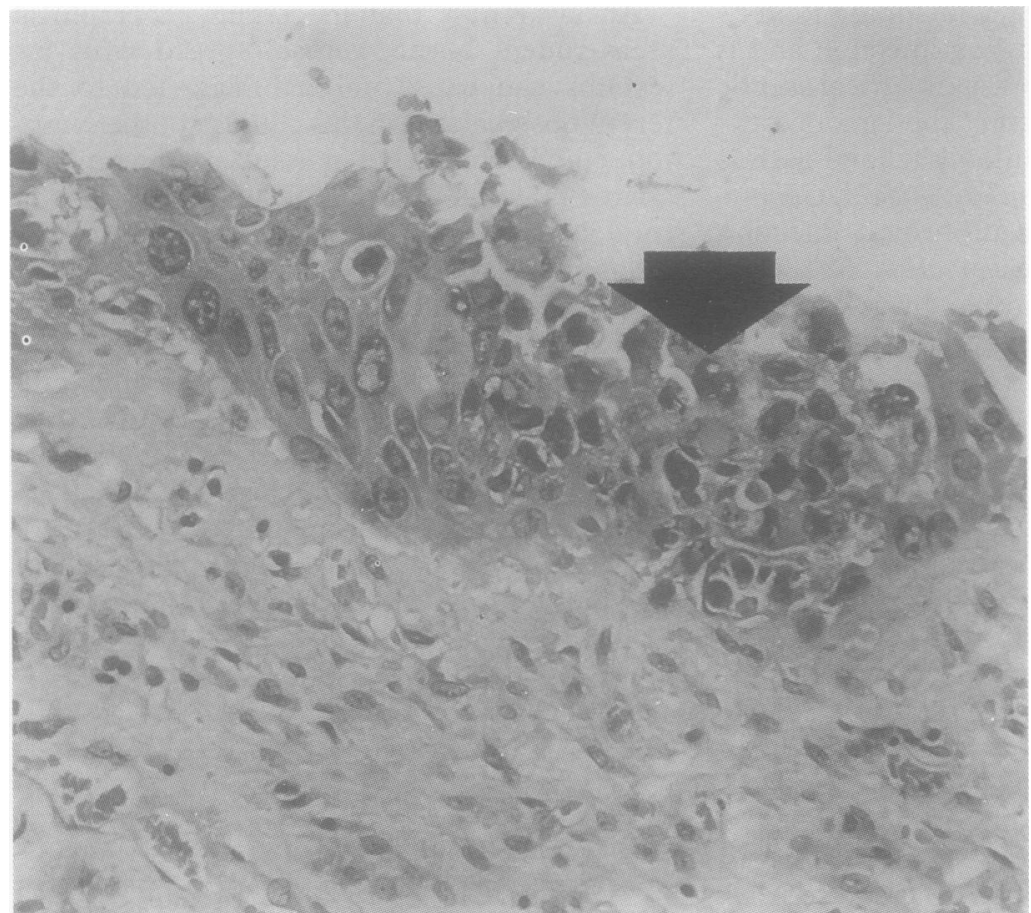

Figure 1 Reactive squamous tracheal epithelium showing several intranuclear inclusions (arrow). Haematoxylin and eosin.

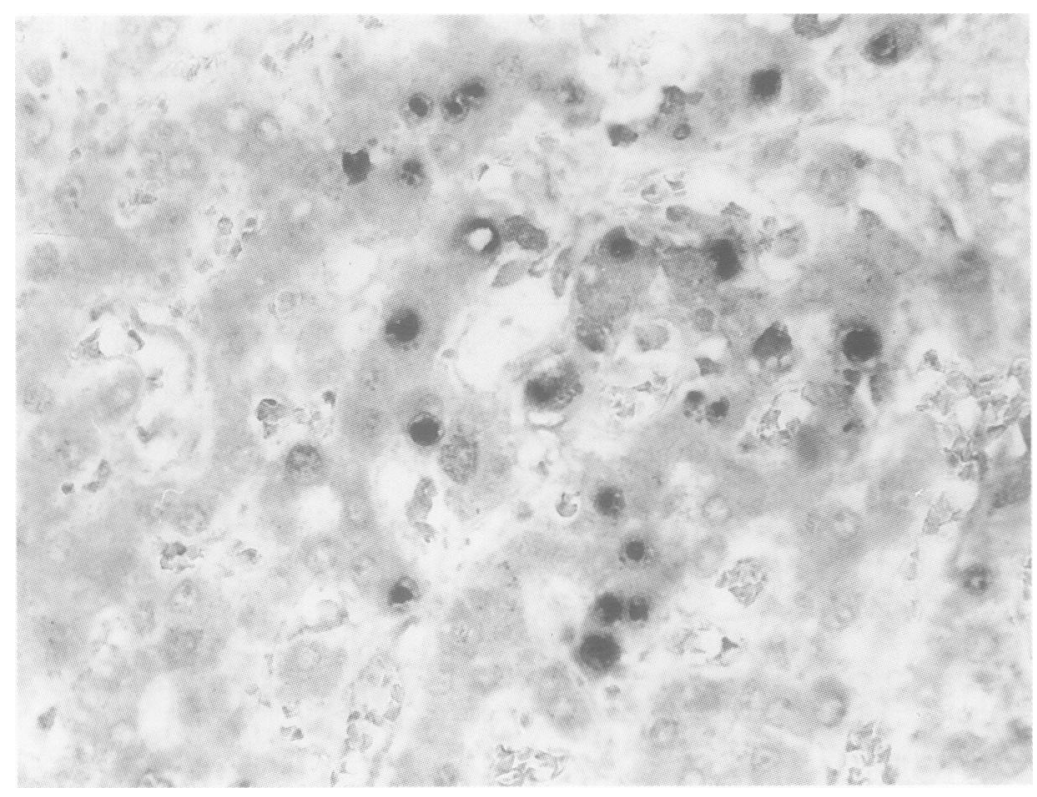

Figure 2 In situ hybridisation demonstrating numerous intranuclear inclusions in the liver, clearly highlighted by the probe.

volvement, ${ }^{1}$ tubulointerstitial nephritis ${ }^{7}$ and haemorrhagic cystitis, ${ }^{8}$ and a case of meningoencephalitis. ${ }^{9}$

In situ hybridisation permits the identification of specific viruses in the tissue sections and has been used for detecting adenovirus infection. ${ }^{10}$ This technique was used to confirm the nature of the intranuclear viral inclusions seen with routine histological stains.

\section{Case report}

The patient was a male infant in whom infantile osteopetrosis was diagnosed at the age of four months with evidence of hydrocephalus and optic atrophy. He received a bone marrow transplant when eight months old and was treated with the immunosuppressive agents cyclosporin A and Campath. After transplantation, the patient had an episode of diarrhoea, when adenovirus was cultured from the faeces, and several episodes of febrile neutropenia including Staphylococcus aureus and haemolytic streptococcal bacteraemias. Six days before he died, the patient developed an ileus, which was managed conservatively. Gram positive cocci were cultured from blood. Four days later the patient developed respiratory distress and required ventilation. $\mathrm{He}$ deteriorated with refractory hypotension and died.

\section{Postmortem findings}

At necropsy, the lungs were firm, heavy and oedematous with petechial haemorrhages. There were ulcers in the ileum, the liver was flabby and yellow, and there was mild ascites. The bones were solid and there was increased cerebrospinal fluid in the subarachnoid space around the brain. The remaining organs were macroscopically normal. Histologically, the lungs showed changes consistent with adult type respiratory distress syndrome, with hyaline membranes, intra-alveolar macrophages and organising exudate. The larynx was ulcerated and occasional "brick-like" intranuclear inclusions were seen in the squamous epithelium and adjacent glands (fig 1).

In the liver there was extensive necrosis involving both periportal and centrilobular areas. Many hepatocytes contained eosinophilic intranuclear inclusions. The ileum showed mucosal ulceration, some villous atrophy, and intranuclear inclusions were seen in the superficial epithelium and in adjacent crypts which showed regenerative changes. Inclusions were also occasionally present in stromal cells in the submucosa deep within the ulceration. Similar inclusions were seen in the appendix. The inclusions seen in the larynx, liver and bowel were typical of those described in adenovirus infections.

Focal ischaemic changes were seen in the myocardium. The bones showed histological changes consistent with osteopetrosis. Little bone marrow engraftment was seen.

\section{In situ hybridisation}

A biotinylated DNA probe for adenovirus (Enzo Diagnostics, New York, USA) was hybridised to the postmortem, paraffin wax embedded tissues, using standard techniques. Hybridised probe was detected using Streptavidin complexed with alkaline phosphatase, with NBT-BCIP as the chromogen (Kreatech Biotin detection system). The slides were counterstained with neutral red. An appendix with adenovirus inclusions served as a positive control.

The probe produced a reactive product in all cells showing intranuclear inclusions on light microscopy, as well as in some adjacent epithelial cells in hepatocytes (fig 2), trachea and bowel. The stromal cells with intranuclear in- 
clusions in the submucosa of the ulcerated ileum were also positive. Occasional epithelial cells were positive in the lungs and bile ducts.

\section{Virology}

Adenovirus was cultured from routine clinical samples and also from postmortem samples of lung and small intestine. The virus was identified as type 32 by neutralisation of infectivity using hyperimmune guinea-pig antiserum.

\section{Discussion}

Previous reports have confirmed the serious pathogenic potential of adenovirus in immunocompromised transplant recipients. Diarrhoea caused by adenovirus infection in bone marrow transplant recipients is well recognised and needs to be distinguished clinically from graft versus host disease. Many adenovirus types have been recognised in these infections including types $2,5,11,12,31$, and 35 . This is the first report that we are aware of adenovirus type 32 causing similar disease in a bone marrow recipient, although isolation of this serotype has been documented in cases of secondary immunodeficiencies such as lymphoma and AIDS, causing hepatosplenomegaly, encephalitis and gastroenteritis, respectively. ${ }^{1}$ Diarrhoea caused by adenovirus types 40 and 41 is well recognised in the immunologically competent host.

In this case the virus gave rise to bowel symptoms including diarrhoea, a severe hepatitis and probably directly, as well as indirectly through the hepatitis, contributed to the adult type respiratory distress syndrome.

The probe used is reported to be specific for adenovirus types $5,7,11,20,40$, and 41 , but there is considerable homology in the DNA sequences and so other adenovirus types may be detected. Adenovirus should be considered in the differential diagnosis of serious sepsis in the immunocompromised patient, and is not an uncommon infection in these patients. In situ hybridisation offers a quick and sensitive method of viral detection, detecting the virus in cells without obvious nuclear inclusions on histology.

The authors thank Professor J S Lilleyman for referring this patient.

1 Hierholzer JC. Adenoviruses in the immunocompromised host. Clin Microbiol Rev 1992;5:262-74.

$2 \mathrm{Kim}$ KS, Gohd RS. Fatal pneumonia caused by adenovirus type 35. Am ₹ Dis Child 1981;135:473-5.

3 Koneru B, Jaffe R, Esquivel CO, Kunz R, Todo S, Iwatsuki $S$, Starzl TE. Adenoviral infections in pediatric liver transplant recipients. $\mathcal{F} A M A$ 1987;258:489-92.

4 Spencer GD, Shulman HM, Myerson D, Thomas ED, McDonald GB. Diffuse intestinal ulceration after marrow transplantation: A clinicopathologic study of 13 patients. Hum Pathol 1986;17:621-33.

5 Landry ML, Fong CKY, Nedderman K, Solomon L, Hsiung GD. Disseminated adenovirus infection in an immunocompromised host. Pitfalls in diagnosis. Am $7 \mathrm{Med}$ 1987;83:555-9.

6 Niemann TH, Trigg ME, Winick N, Penick GD. Disseminated adenoviral infection presenting as acute pancreatitis. Hum Pathol 1993;24:1145-8.

7 Ito M, Hirabayashi N, Uno Y, Nakayama A, Asai J. Necrotising tubulointerstitial nephritis associated with adenovirus infection. Hum Pathol 1991;22:1225-31.

8 Murphy GF, Wood DP Jr, McRoberts JW, Henslee-Downey PJ. Adenovirus associated hemorrhagic cystitis treated with intravenous Ribavirin. F Urol 1993;149:565-6.

9 Davis D, Henslee PJ, Markesberry WR. Fatal adenovirus meningoencephalitis in a bone marrow transplant patient. Ann Neurol 1988;23:385-9.

10 Porter HJ, Padfield CJH, Peres LC, Hirschowitz L, Berry $\mathrm{PJ}$. Adenovirus and intranuclear inclusions in appendices in intussusception. $\mathcal{f}$ Clin Pathol 1993;46:154-8.

\section{Sudden death from coronary artery dissection}

Department of
Histopathology,
Royal Hampshire
County Hospital,
Winchester
A C Bateman
A C Vincenti
Department of
Histopathology,
Southampton General
Hospital,
Southampton
P J Gallagher
Correspondence to:
Dr A C Bateman,
Level E, South Block,
Southampton General
Hospital,
Tremona Road,
Southampton
SO16 6YD.
Accepted for publication
18 January 1995

\author{
A C Bateman, P J Gallagher, A C Vincenti
}

\begin{abstract}
Spontaneous dissection of the coronary arteries is an uncommon condition that may lead to sudden, fatal coronary artery occlusion. Three cases of sudden death attributable to coronary artery occlusion are presented. Dissection was associated with Marfan's syndrome in the first case, and occurred three weeks postpartum in the second case. In case 1 , dissection occurred within the intima, and was not associated with an inflammatory cell infiltrate. In cases 2 and 3, dissection occurred between the tunica media and the external elastic lamina, and was associated with a mixed
\end{abstract}

inflammatory infiltrate, rich in eosinophils, T lymphocytes, and histiocytes. The spatial limitation of the inflammatory infiltrate to the adventitial compartment, together with the abscence of inflammation in case 1 , suggests a reactive origin rather than a causative role for the inflammatory cells. Detailed examination of serial blocks of any coronary artery occlusion is essential in young patients. ( $\mathcal{H}$ Clin Pathol 1995;48:781-784)

Keywords: Coronary arteries, dissection, sudden death, inflammation. 